\title{
Un nouveau visage du sanctuaire d'Apollon à Délos, ou Délos prend de la hauteur
}

\author{
A new face of the Apollo sanctuary in Délos, \\ or Délos takes on a new dimension
}

\author{
R. Étienne ${ }^{1}$, L. Costa ${ }^{2}$ \\ ${ }^{1}$ Professeur émérite, Université de Paris 1, UMR 7041, roland.etienne4@wanadoo.fr \\ ${ }^{2}$ Ingénieur de recherche, CNRS, UMR 7041, laurent.costa@cnrs.fr
}

\begin{abstract}
RÉSUMÉ. Délos est avec Delphes, l'un des plus prestigieux sanctuaires grecs de la Méditerranée. Utilisé depuis le huitième siècle BC jusqu'à la fin de l'Antiquité romaine, il restera le plus important sanctuaire maritime de la Méditerranée dans l'Antiquité.

Nous présentons ici le travail réalisé sur la maquette numérique du sanctuaire mis en place dans le cadre de la publication du Sanctuaire. Outre la synthèse qu'elle nous a permis de réaliser, la démarche de restitution 3D a été l'occasion d'interroger nos visions de ce patrimoine majeur et de proposer de nouveaux angles de vue et de compréhension de ce sanctuaire. La démarche illustre de manière assez explicite tout l'intérêt du développement d'une approche numérique intégrée allant de la collecte jusqu'à la restitution et démontre l'intérêt heuristique des restitutions numériques. Ce travail débouche sur une réflexion liée à l'objectivité des restitutions de ces espaces anciens. Ici sur Délos, par exemple, la restitution des colonnes ou des bases portant les statues transforme la vision que nous pourrions avoir de la zone autour de l'autel d'Apollon. Ils permettent de percevoir l'obstruction du sanctuaire vers 150 av. J.-C. qui ne pouvait pas être connue avant de faire la restitution.

ABSTRACT. Delos is with Delphes, one of the most prestigious Greek sanctuaries in the Mediterranean. Used from the eighth century BC until the end of Roman antiquity, it will remain the most important maritime sanctuary in the Mediterranean in antiquity.

We present here the work done on the digital model of the sanctuary set up as part of the publication of the Sanctuary. In addition to the synthesis that it has enabled us to achieve, the 3D restitution process has been an opportunity to question our visions of this major heritage and to propose new angles of view and understanding of this sanctuary. The approach illustrates quite explicitly the value of developing an integrated digital approach from collection to restitution and demonstrates the heuristic value of digital restitution. This work leads to a reflection linked to the objectivity of the restitution of these ancient spaces. Here in Delos, for example, the restitution of the columns or bases bearing the statues transforms the vision we might have of the area around the altar of Apollo. They make it possible to perceive the obstruction of the sanctuary around $150 \mathrm{BC}$ which could not be known before making the restitution.
\end{abstract}

MOTS-CLÉS. Délos, sanctuaire, 3D, Restitution.

KEYWORDS. Delos, sanctuary, 3D, restitution.

Nous travaillons depuis dix ans sur le sanctuaire d'Apollon à Délos et la nouvelle publication rend compte du résultat de nos recherches d'équipe ${ }^{1}$. Même un bon connaisseur des réalités déliennes

\footnotetext{
${ }^{1}$ R. ÉTIENNE (dir.), Le Sanctuaire d'Apollon à Délos, T. I Architecture, topographie, histoire, Exploration archéologique de Délos 44 (2018). TII (sous presse) Les petits monuments (Fr. Herbin).
}

Ont pris part à la publication : Introduction : des fouilles et des plans (R.Étienne, L. Costa),

Première partie, Analyse des espaces et des bâtiments, Ch. I Le sanctuaire d'Apollon : extension et limites (R.Étienne), Ch. II Géomorphologie, hydrologie et littoral du Sanctuaire (St. Desuelles, Cl. Hasenohr), Ch. III Les périboles du Sanctuaire d'Apollon (R.Étienne), Ch. IV Les Propylées et autres entrées (R.Étienne, M. Wurch Kozelj), Ch. V Le Bouleutérion (édifice $\Delta$ ) (R.Étienne, J.P. Braun), Ch. VI Le Pôrinos naos (R.Étienne, J.P. Braun), Ch. VII Disposition spatiale des offrandes monumentales et circulations dans le Sanctuaire (Fr. Herbin), Ch VIII Édifices et constructions des époques impérialeet protobyzantine dans le Sanctuaire d’Apollon (E. Le Quéré). 
pourrait à juste titre se demander s'il y avait quelque chose de neuf à trouver après 150 ans de fouilles grecques et françaises sur ce site. En fait, il fallait combler de nombreuses lacunes dont le détail apparait clairement dans la publication : disons que le programme de la «Grande fouille » du début du XXe s. n'avait été que partiellement rempli ${ }^{2}$ et qu'il restait des monuments à publier, le tracé des périboles à préciser, la ligne de rivage à fixer... Les études antérieures avaient fait la part belle aux études monographiques de monuments, aux catalogues divers d'inscriptions, de céramique ou de statues, mais il n'existait pas de synthèse et manquaient notamment des plans historiques permettant de saisir le développement du sanctuaire d'Apollon des origines à la fin de l'Antiquité. C'est ce à quoi nous nous sommes attachés et nous allons présenter les résultats en nous servant des images en 3D auxquelles nous avons abouti. Les figures 1 et 2 permettent de rendre compte de l'écart entre les réalités de terrain et les restitutions.

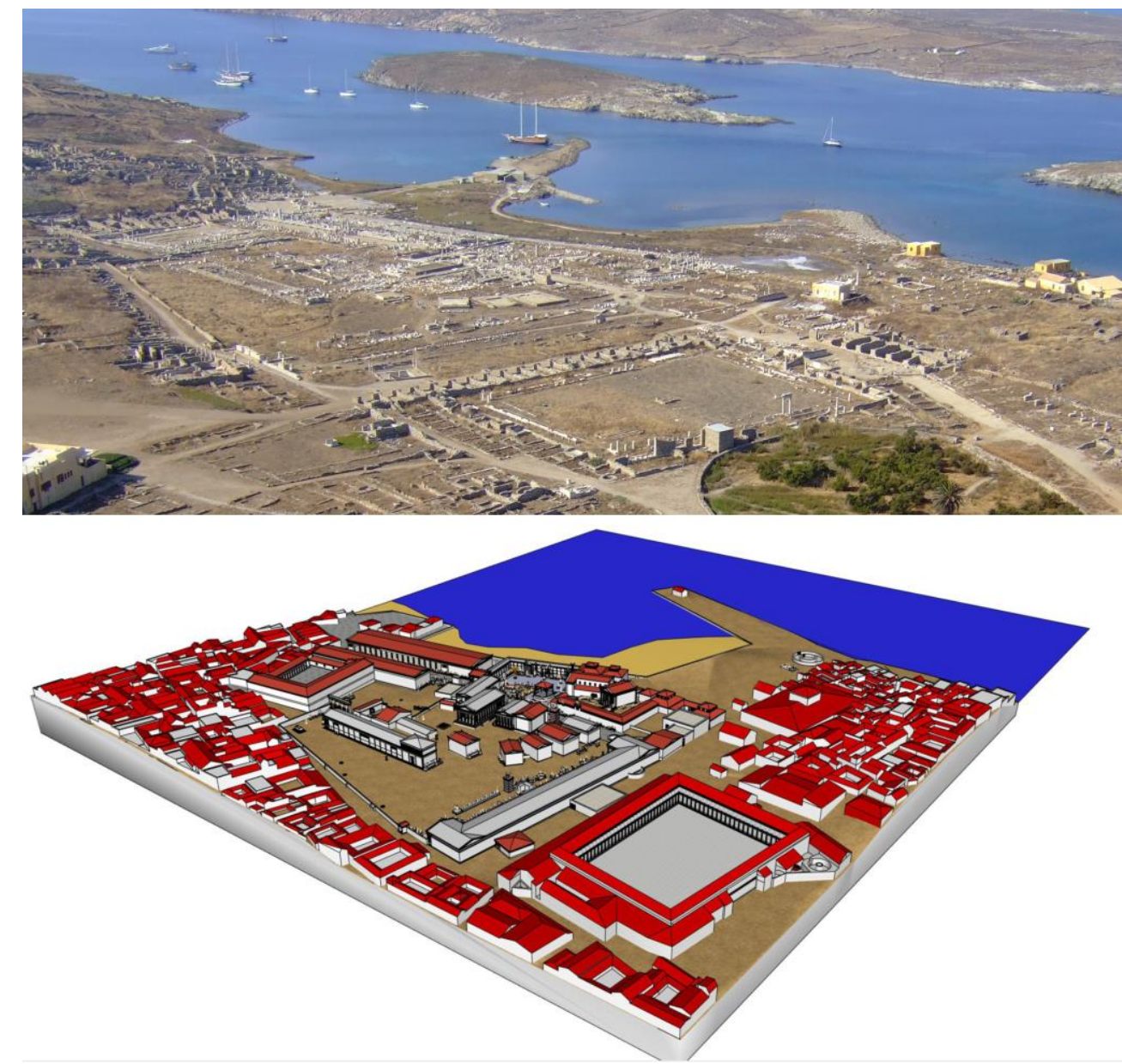

Figure 1. Délos, Rhénée, vue prise du Nord-Est (cl. Chr. Gaston, 2006). En bas, restitution du sanctuaire d'Apollon vers 100 av. J.-C., selon la même orientation.

Deuxième partie : Le développement historique, Ch. IX Origines du Sanctuaire d'Apollon (Fr. Prost), Ch. X Le sanctuaire d'Apollon au $\mathrm{VII}^{\mathrm{e}}$ et $\mathrm{VI}{ }^{\mathrm{e}}$ siècles (Fr. Prost), Ch. XI Un sanctuaire atticisé : $\mathrm{V}^{\mathrm{e}} \mathrm{I} \mathrm{V}^{\mathrm{e}}$ siècles (R. Étienne), Ch. XII L'indépendance et la monarchisation du Sanctuaire d'Apollon (314 à 168/7 av. J.-C.) (R. Étienne), Ch. XIII Le retour des Athéniens : le Sanctuaire du Ile siècle à 88 av. J.-C., Ch. XIV Un paysage sacré en mutation du I ${ }^{\mathrm{er}} \mathrm{s}$. av. J.-C. à la fin de l'époque impériale (E. Le Quéré)

Troisième partie : Appendices, Ch. XV Les bâtiments du Hiéron (R. Étienne), Ch. XVI Puits et canalisations (L. Chevalier), Ch. XVII Retour sur la céramique à décor géométrique du Hiéron (Th. Brisart).

Conclusion : A propos des images 3D (L.Costa, R. Étienne)

Cette recherche a été financée par l'École française d'Athènes et l'UMR 7041 (Arscan).

2 On entend par 'Grande fouille' les travaux qui se sont déroulés à Délos à partir de 1903-1904 sur une grande échelle grâce au mécénat du duc de Loubat, philanthrope franco-américain, sous l'impulsion de M. Holleaux, directeur de l'École française, qui s'était fixé pour but d'achever entre autre le déblaiement du sanctuaire et qui réunit pour ce faire une équipe pluridisciplinaire. 


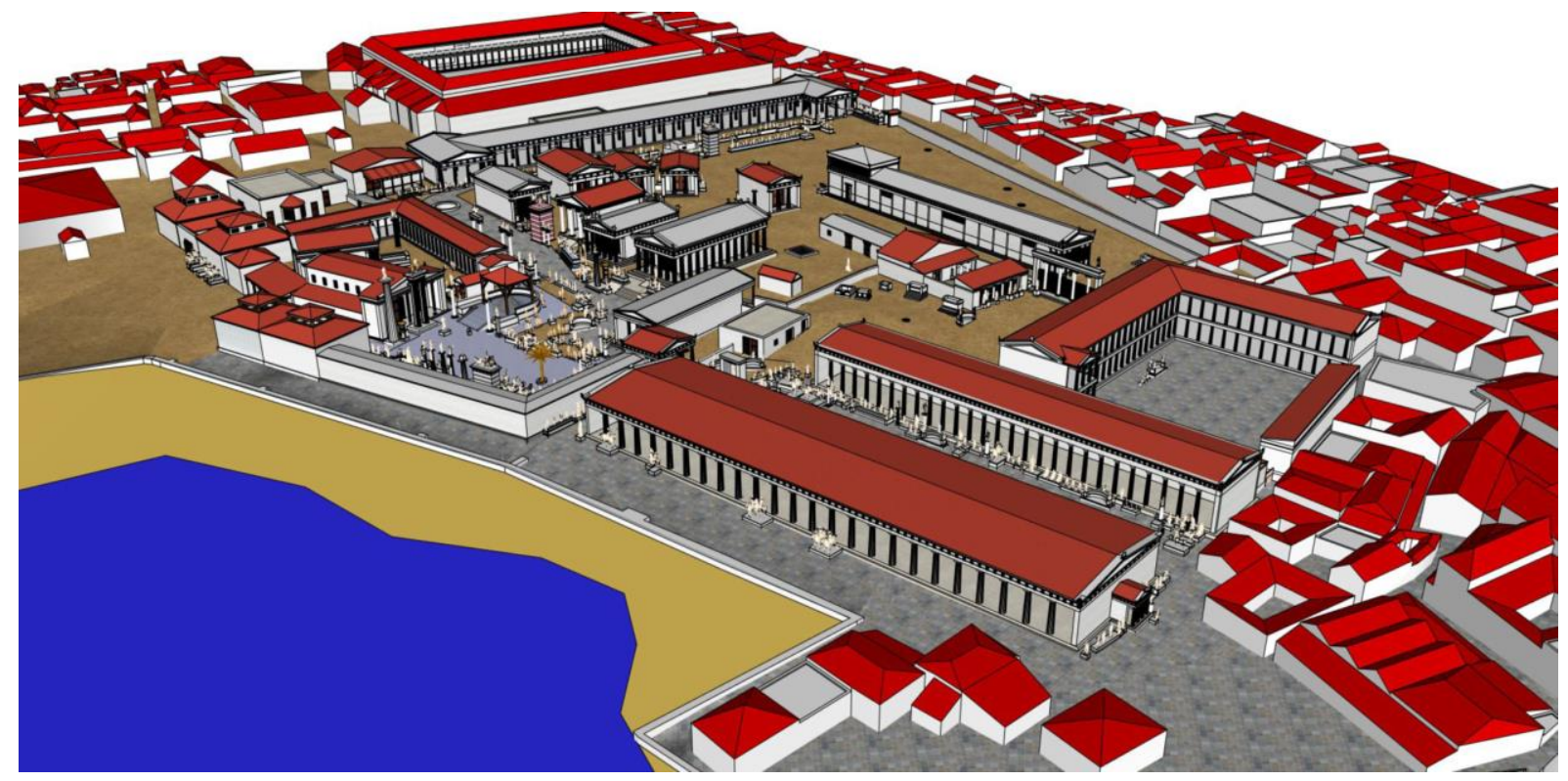

Figure 2. Le sanctuaire d'Apollon vers 100 av. J.-C., vu du Sud-Ouest.

Nous suivrons le parcours d'un sacrifice au temps de la grande prospérité délienne, au II ${ }^{\mathrm{e}}$ s. av. J.-C. Les processions en l'honneur du dieu Apollon empruntaient la voie sacrée qui partait du port actuel, passait entre les deux portiques, celui de l'Est et celui de l'Ouest dédié par le roi Philippe V de Macédoine qui avait fait graver son nom sur l'architrave (figure 3).

Ce qui est vraiment nouveau c'est la restitution des statues et autres petits monuments qui se trouvaient en avant des portiques. Il ne s'agit pas d'une restitution arbitraire, car nous avons étudié toutes les bases et restitué leur élévation ainsi que les statues ou groupes statuaires qu'elles portent avec autant de précision que possible ${ }^{3}$.

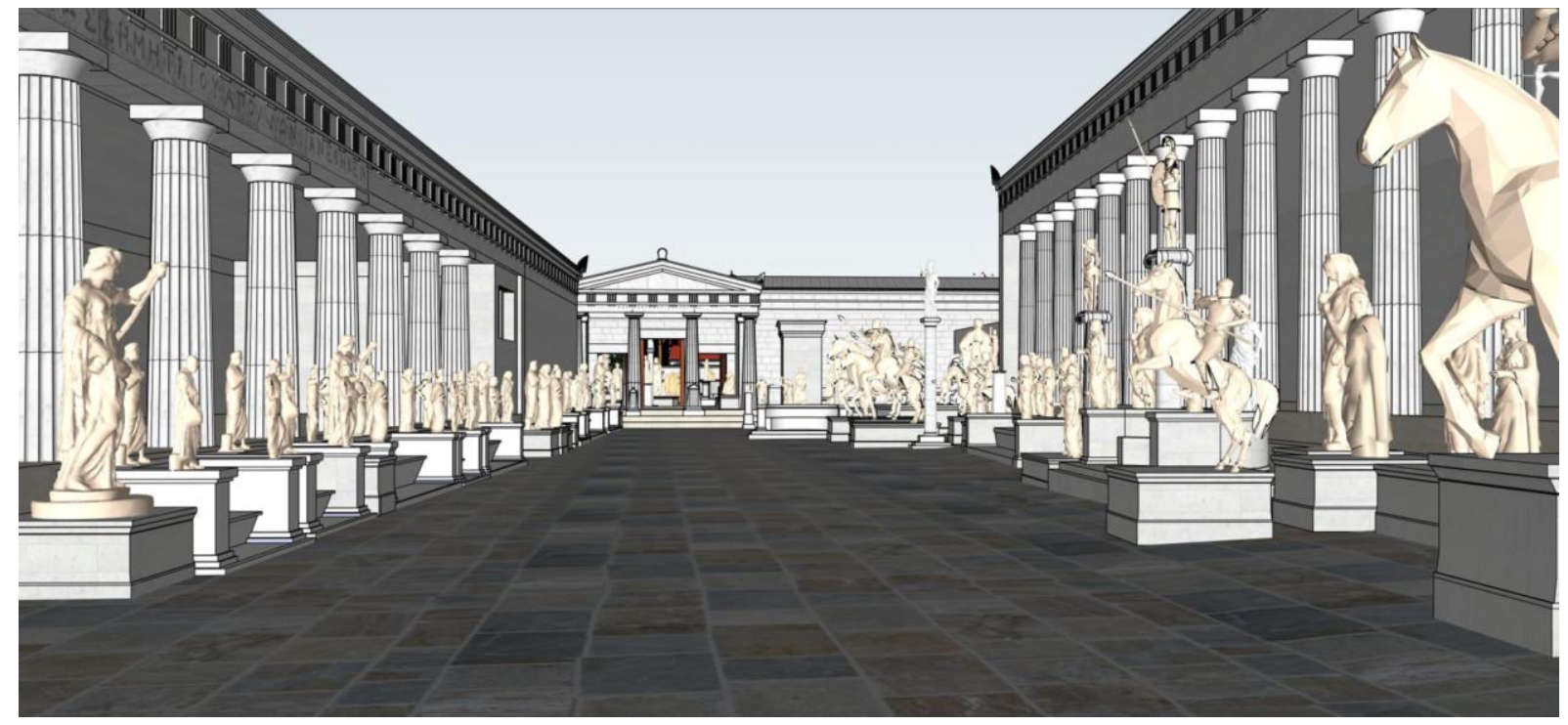

Figure 3. La voie sacrée entre les deux portiques ; à gauche, portique de Philippe $V$ et, au fond, les Propylées.

\footnotetext{
${ }^{3}$ Les bases en front du portique de Philippe ont été étudiées par R. VALLoIS, Le portique de Philippe, EAD VII 1 (1923), celles de l'autre côté de la voie sacrée sont en cours d'étude.
} 
On peut imaginer l'extraordinaire défilé avec les bêtes de sacrifice aux cornes ornées d'or: une inscription rappelle qu'une des bêtes offertes par les gens de Beyrouth portait un panneau sur lequel était inscrit le nom d'un Romain, Marcus Minatius , bienfaiteur de la communauté des Levantins.

Le cortège aboutissait aux grands Propylées (figure 4), que l'on peut restituer jusqu'à la toiture. On a des difficultés pour retrouver les traces de l'entrée à l'époque archaïque, mais les Propylées hellénistiques sont eux très bien connus. Ces propylées ont été construits après 167 av. J.-C. par les Athéniens qui ont gravé leur nom sur l'architrave pour marquer qu'ils étaient redevenus les maîtres du lieu, après avoir dû restituer l'île et la gestion du sanctuaire aux Déliens, à la fin du $\mathrm{IV}^{\mathrm{e}} \mathrm{s}$. (314 av. J.C.). Le plan est le premier de ce type avec quatre colonnes côté extérieur et deux colonnes in antis (figure 5), du côté du sanctuaire. Le bâtiment étant tout en marbre, et doté d'un escalier, il faut croire que l'on installait un passage en bois pour permettre aux bêtes d'accéder sans difficulté au cœur du sanctuaire, sans abîmer la construction (rappelons que c'était à certains moments une centaine de bêtes qui entraient pour être sacrifiées).

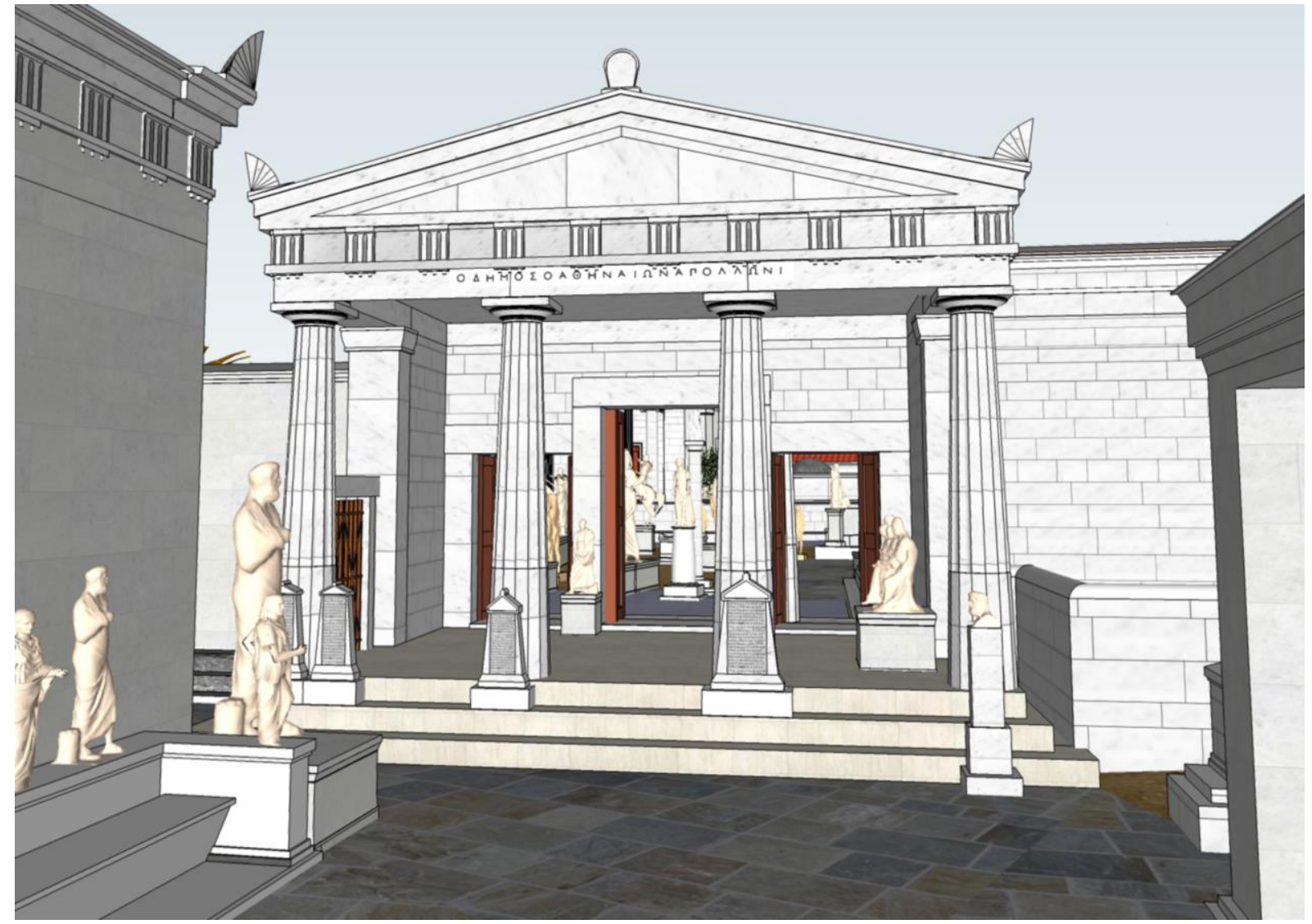

Figure 4. Les Grands Propylées, au milieu du Irr s. av. J-C., façade Sud. 


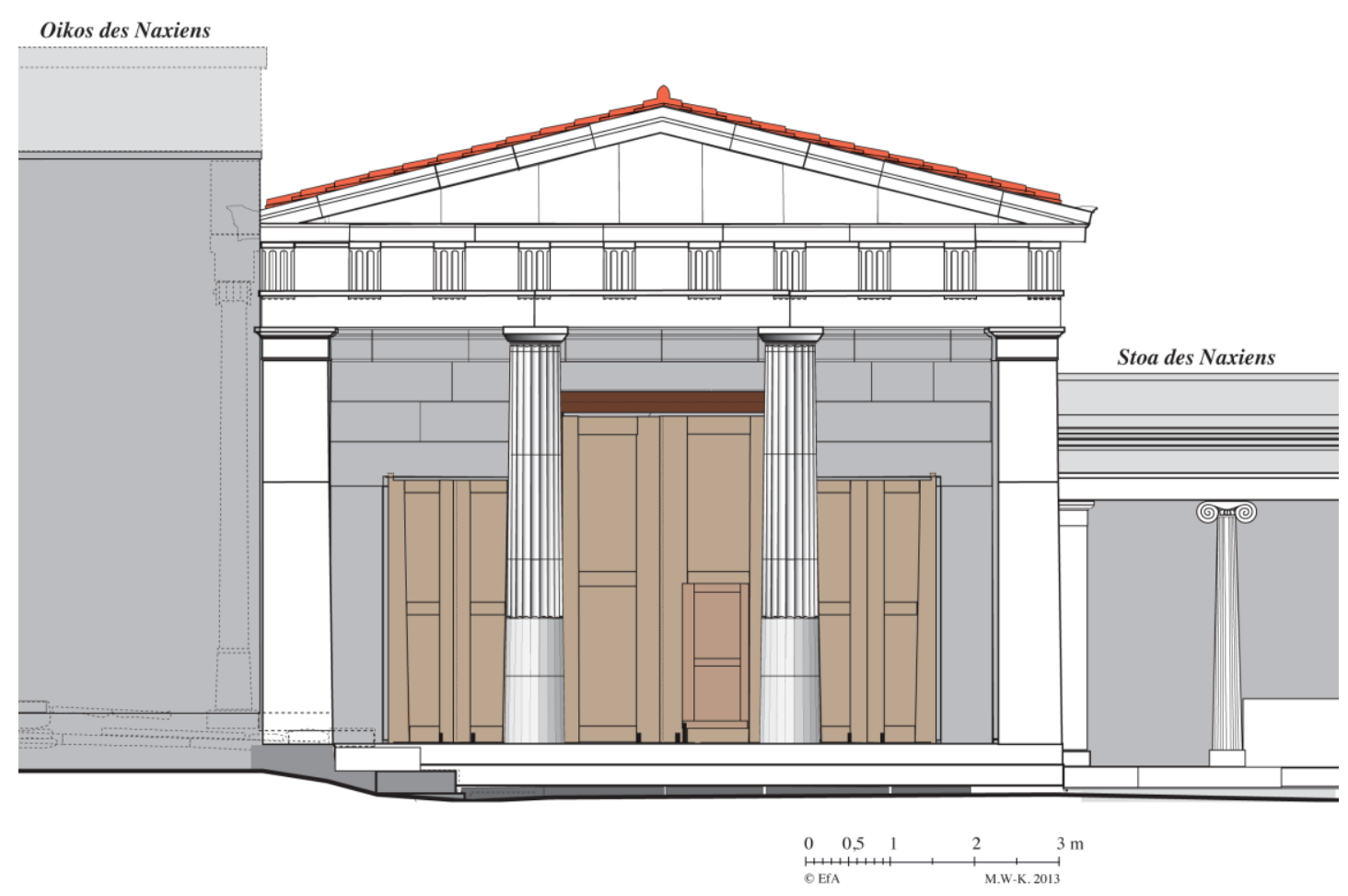

Figure 5. Les Propylées côté Nord, entre l'Oikos des Naxiens, à gauche, et la Stoa des Naxiens, à droite (étude R. Étienne, M. Wurch-Kozelj).

Après avoir passé les Propylées que l'on fermait la nuit avec de solides portes, qui empêchaient que les bêtes (les chiens d'après une loi sacrée) n'entrent dans l'espace sacré et que les hommes n'y passent la nuit, comme le dit une inscription. Au-delà on découvrait le cœur du sanctuaire. A l'époque où nous nous plaçons il était très encombré par les monuments et le spectacle était impressionnant (figure 6). De la droite vers la gauche : le palmier en bronze offert par l'Athénien Nicias à la fin du $\mathrm{V}^{\mathrm{e}} \mathrm{s}$. (c'était l'homme le plus riche d'Athènes et il dirigea l'expédition athénienne en Sicile en 415) sépare les colonnes des Propylées, à droite, des quatre temples d'Apollon : l'Oikos des Naxiens, avec deux colonnes in antis et, plus au Nord, les trois autres temples d'Apollon construits au $\mathrm{V}^{\mathrm{e}} \mathrm{s}$. Jusque là, il n'y a guère de surprise, c'est la restitution du paysage délien habituel. De même, le bâtiment recouvert d'un baldaquin et d'un toit n'est autre que le grand autel d'Apollon autour duquel on rangeait les bêtes en rangs serrés avant le sacrifice (l'espace devant l'autel ne mesure que $415 \mathrm{~m} 2$, ce qui semble bien réduit pour y loger une hécatombe, cent bêtes et les sacrificateurs). En revanche les colonnes votives surmontées de statues sont un des apports intéressants des dernières recherches.

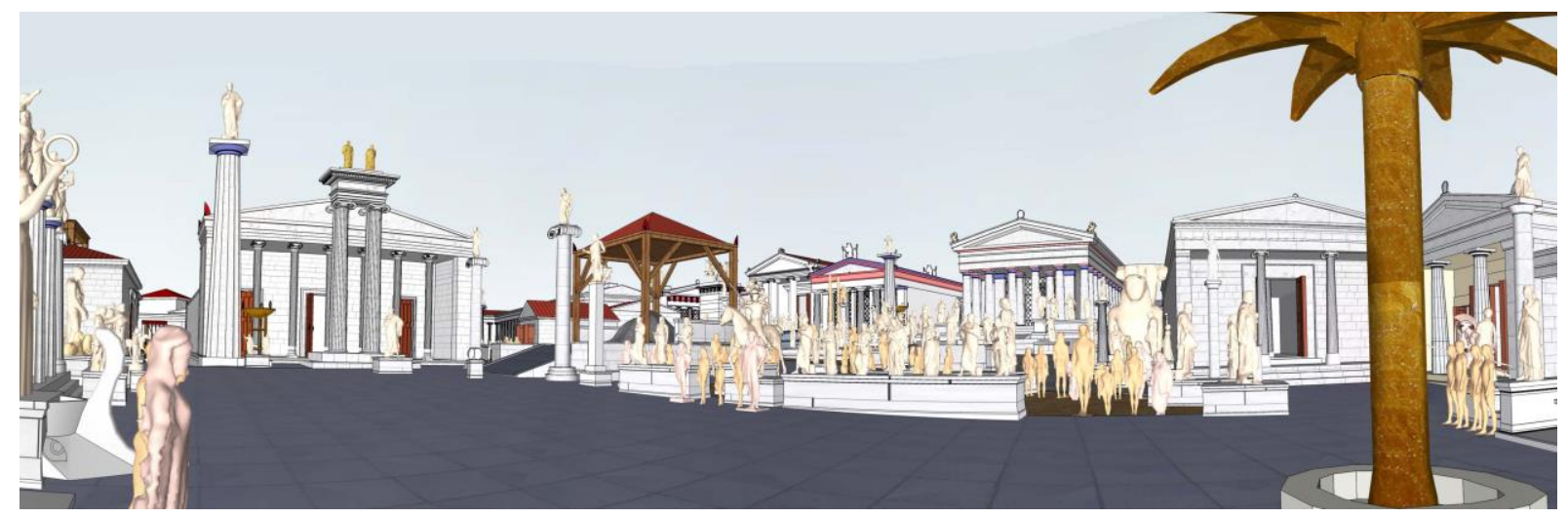

Figure 6. Le sanctuaire vu des Propylées, vers 100 av.J.-C. (à droite, le palmier en bronze de Nicias). 


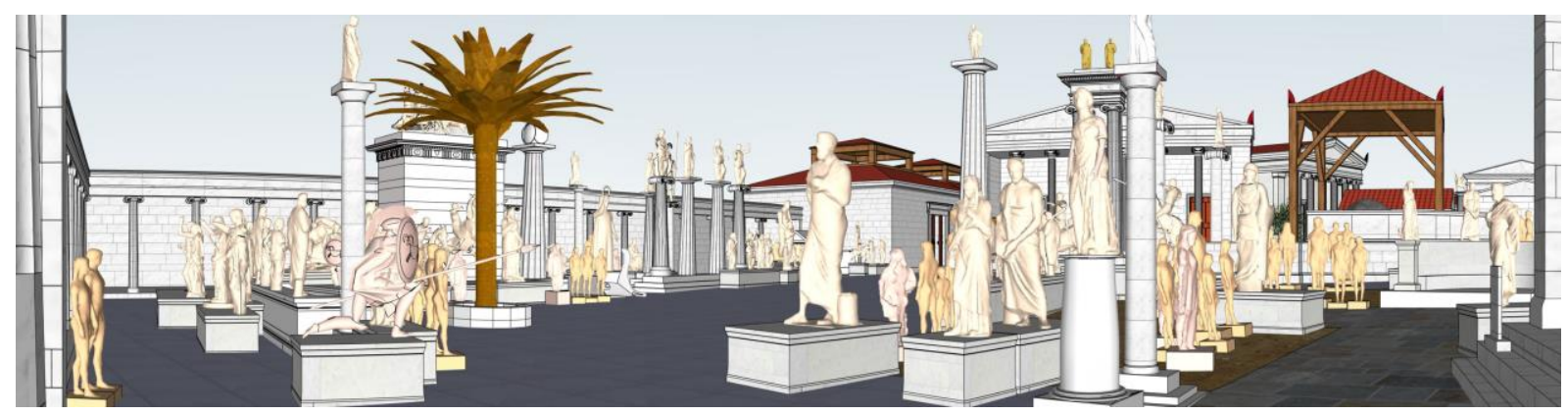

Figure 7. Le sanctuaire côté Est, à droite le palmier de Nicias et les Propylées.

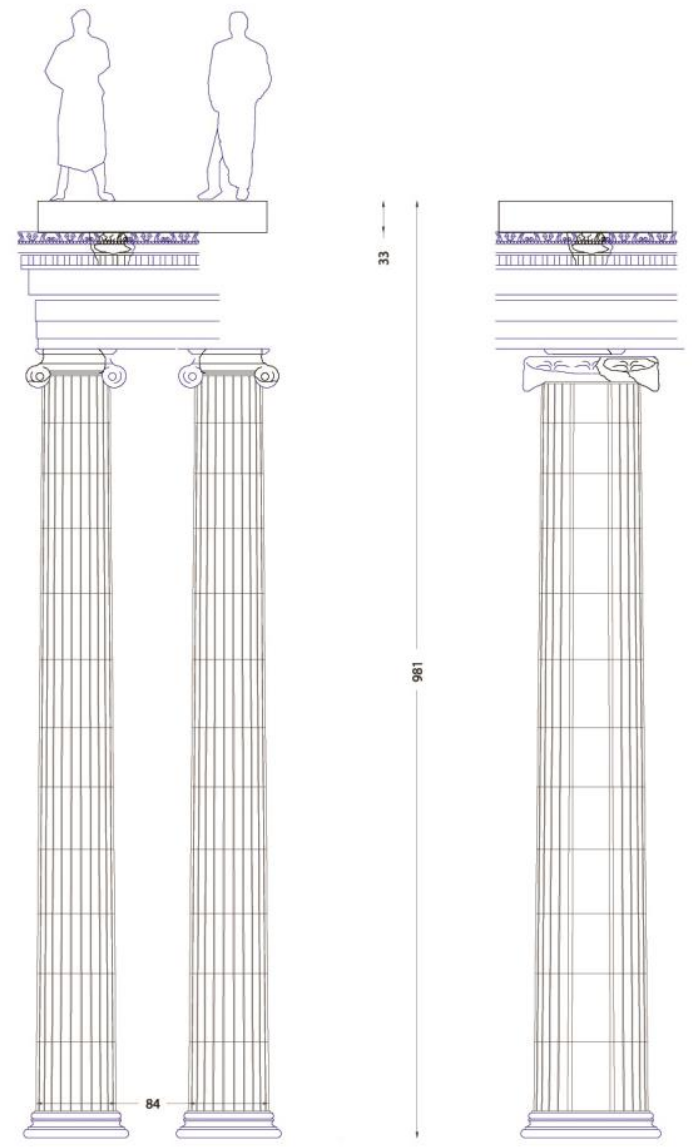

Figure 8. Monument à deux colonnes portant deux statues royales, dont celle d'Alexandre le Grand (étude Fr. Herbin, J.-P. Braun).

L'identification de 20 colonnes votives transforme le paysage du sanctuaire tel que l'on pouvait le restituer (figure 7). Chaque roi voulait avoir sa statue-portrait posée aussi haut que celle de ses collègues. On peut considérer comme un gain important pour la topographie du sanctuaire la colonne que nous replaçons dans l'axe du Pythion et qui mesurait $10 \mathrm{~m}$ de haut (figure 8) : nous lui avons attribué un bloc d'architrave portant une dédicace à Alexandre le grand, dont le nom est lisible, et à un autre souverain qui avait fait la dédicace du monument; son nom a malheureusement disparu, mais l'énorme fondation en granit taillé qui la portait doit appartenir au début du III $^{\mathrm{e}}$ s., comme d'autres bâtiments mieux datés utilisant la même technique. La colonne est placée dans l'axe du temple construit au $\mathrm{IV}^{\mathrm{e}}$ s. par les Athéniens (Pythion) et nous pensons qu'il s'agissait de le cacher en partie pour attirer l'attention sur les rois, nouveaux maîtres de l'île. Polybe nous signale qu'autour de l'autel d'Apollon, Antiochos IV vers 160 avait installé les membres de sa famille. On a imaginé des solutions multiples sur la place de ces statues; elles occupaient sans nul doute une place sur des colonnes proches de l'autel. 


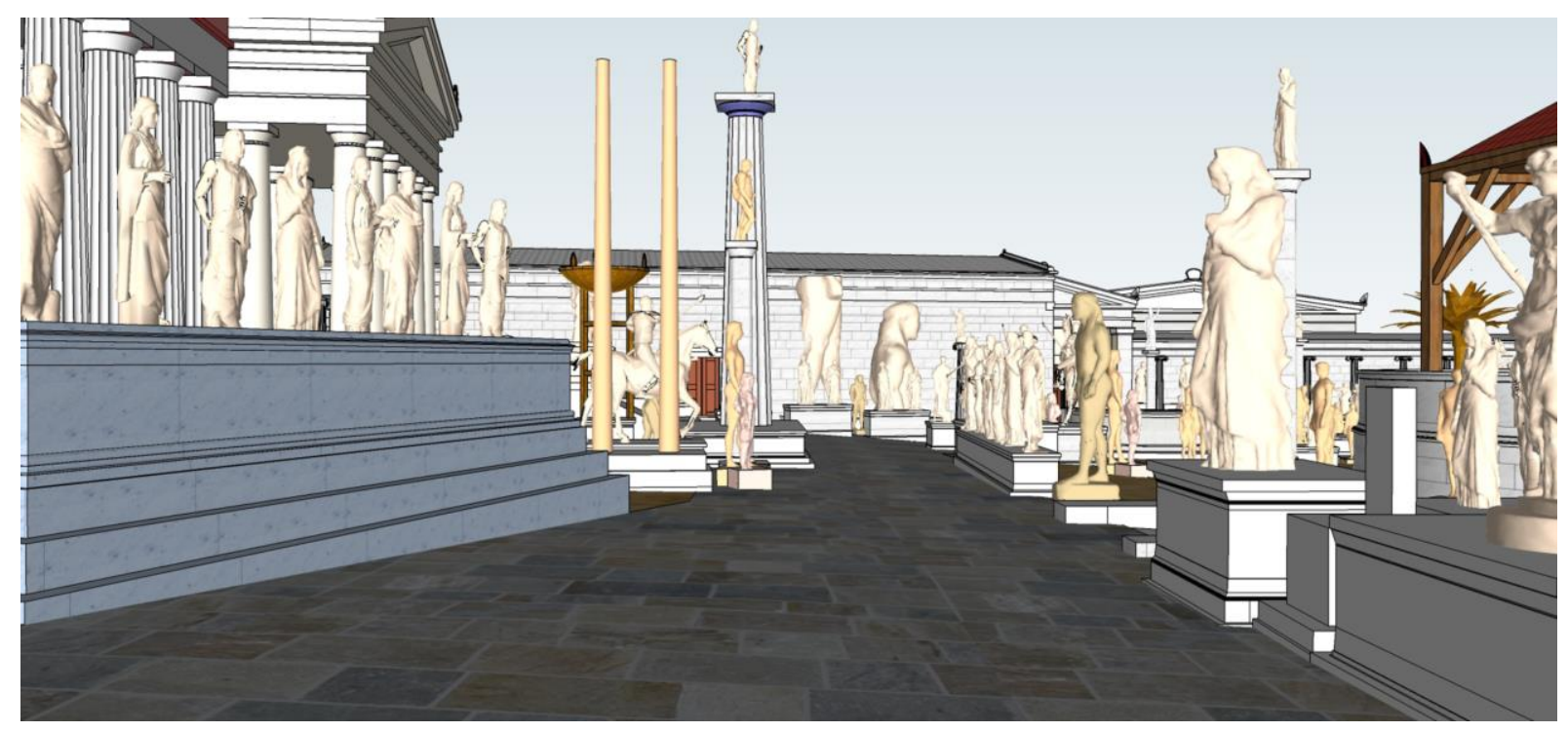

Figure 9. Monuments bordant la voie passant devant les temples (au fond, Kouros des Naxiens en deux parties).

Le cheminement des pélerins, au-delà de l'autel, passait le long de la voie qui longeait le côté Nord de l'Oikos des Naxiens et passait devant le colosse des Naxiens. La présentation de cette statue en deux parties mérite que l'on s'y arrête (figure 9). Le palmier de bronze dédié par le stratège Nicias à la fin $\mathrm{du} \mathrm{V}^{\mathrm{e}}$ s., dont nous avons déjà parlé, était tombé dessus et l'avait brisée. Cette historiette rapportée par Plutarque a fait couler beaucoup d'encre, car la base du colosse est trop loin aujourd'hui du palmier et s'il en était de même dans l'Antiquité, le palmier n'a pu briser le Colosse en tombant (fig. 10). Les études récentes montrent :

- que la base a dû être déplacée dans l'Antiquité et placée du côté des temples (figure 9) ;

- que le colosse était présenté en deux morceaux, ce qui est pour nous surprenant. Et ce n'est pas le seul exemple, car on a mis en évidence la présentation d'un kouros brisé près du Trésor 5 (ajoutons que le sanctuaire était périodiquement nettoyé, raison pour laquelle les statues du $\mathrm{VII}^{\mathrm{e}} \mathrm{s}$. se retrouvent dans le massif de fondation de la stoa des Naxiens).
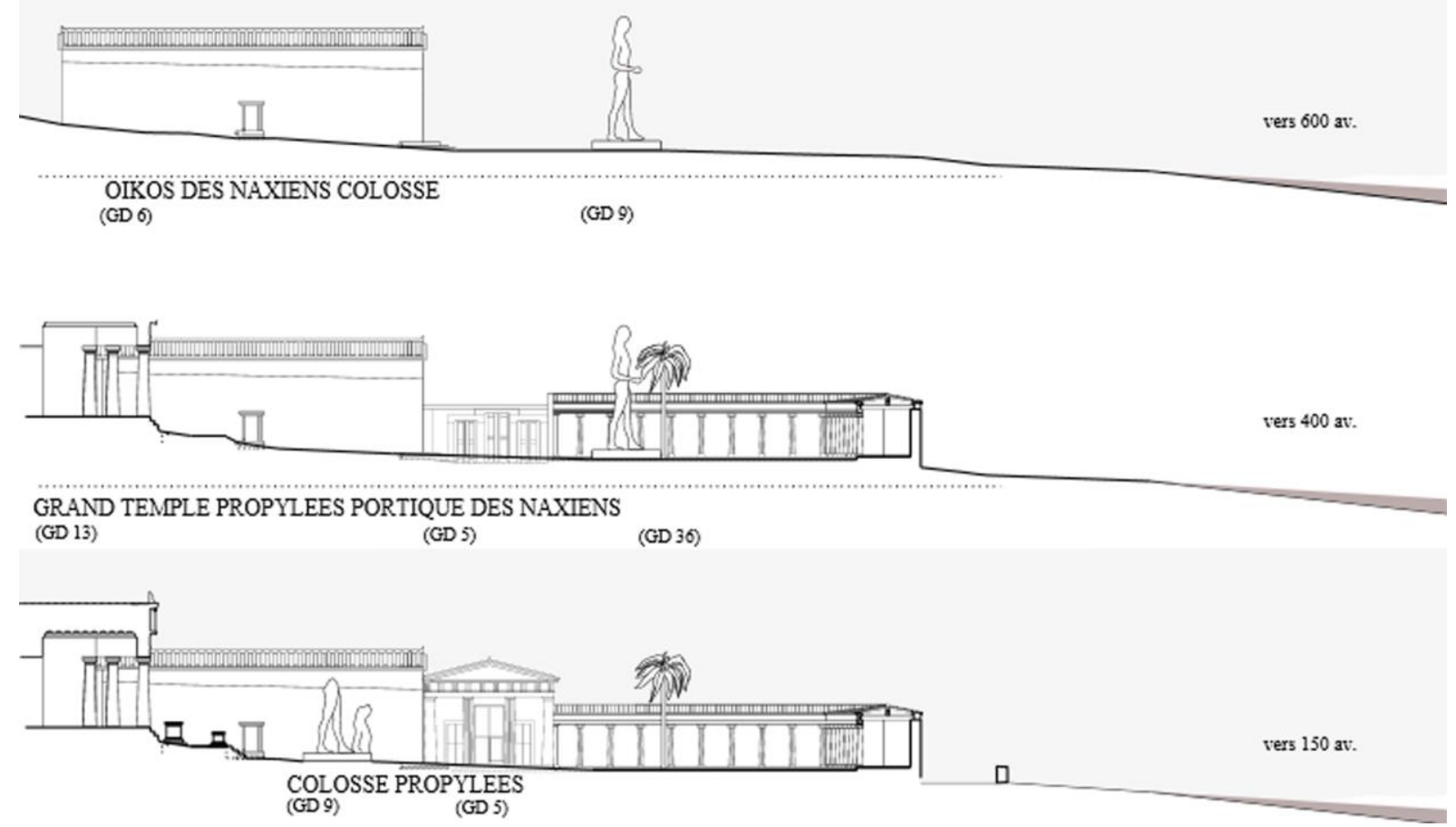

Figure 10. Les avatars du kouros des Naxiens (dessin J-P. Braun). 
Il ne s'agit que d'un détail, mais il oblige à nuancer une vision trop idyllique d'un sanctuaire qui, par certains aspects, se présentait comme un «capharnaüm»d'offrandes de toutes époques et plus ou moins bien conservées.

Le pèlerin pouvait continuer sa visite vers le Nord en passant devant les trois temples (figure 11) ; signalons que nous restituons maintenant le temple dit Temple en calcaire de la fin du VIe s. avec quatre colonnes ioniques prostyles, que l'on aperçoit derrière le monument en marbre rose. L'œil était attiré par deux monuments importants, l'un portant les statues de la dynastie de Pergame en marbre bleu, et l'autre un pilier en marbre rose qui pouvait supporter un char conduit par un souverain de Pergame, selon les propositions de Fr. Herbin.

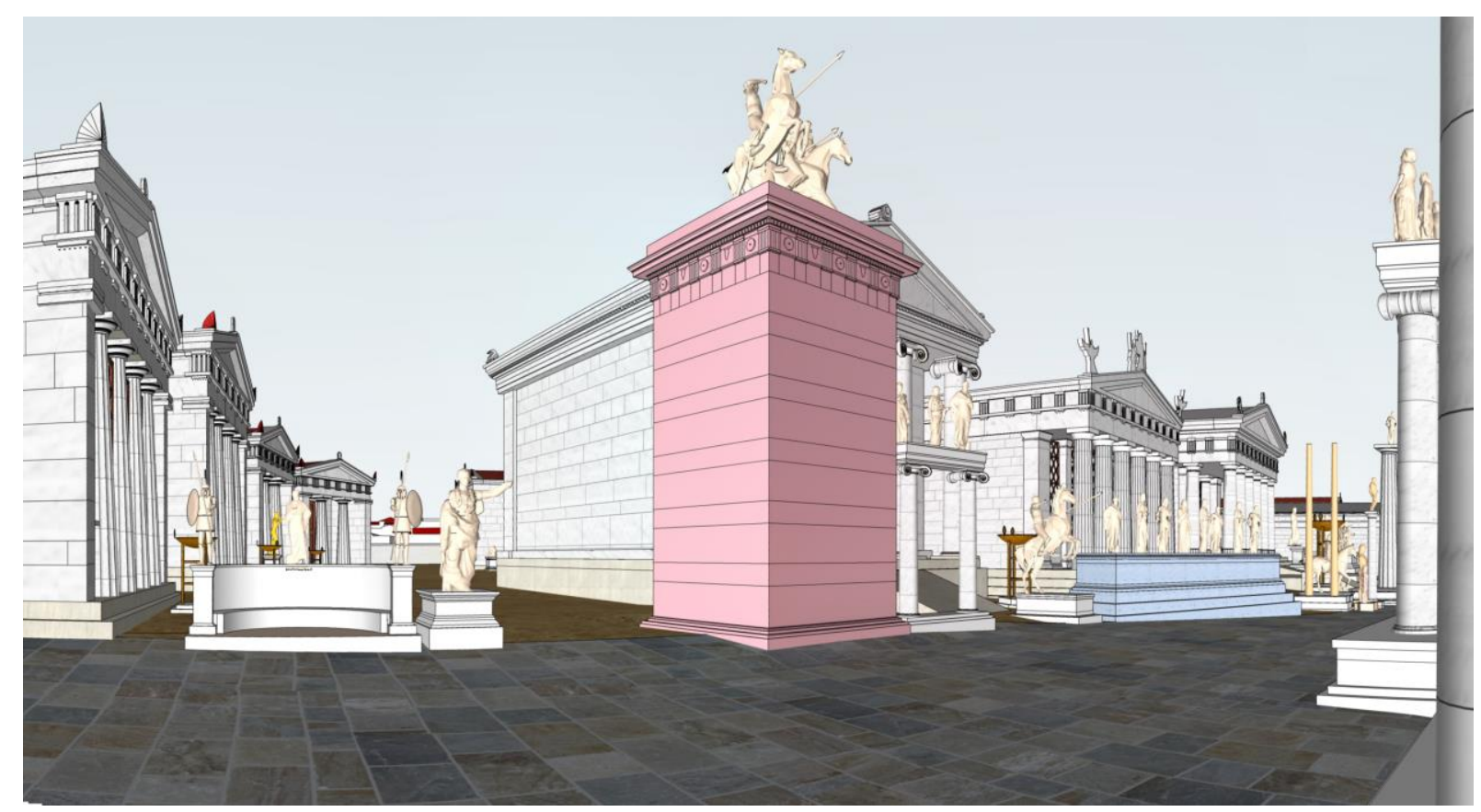

Figure 11. Monuments pergaméniens en marbre rose et bleu (étude Fr. Herbin) ; à gauche façades des Trésors $1-5$ et à droite façades des temples.

Les couleurs ne sont donc pas choisies au hasard, mais correspondent à la réalité. C'est le cas aussi du décor du long portique Nord, dédié par le roi Antigone Gonatas vers 250 et qui portait des têtes de bœuf sur les triglyphes (figure 12). Ces têtes de taureau sont un symbole de la dynastie macédonienne que l'on retrouve comme ornement des colonnes du bâtiment qui contenait un bateau dédié par Démétrios Poliorcète en souvenir de la bataille de Salamine de Chypre en 306, grande victoire sur les Lagides.

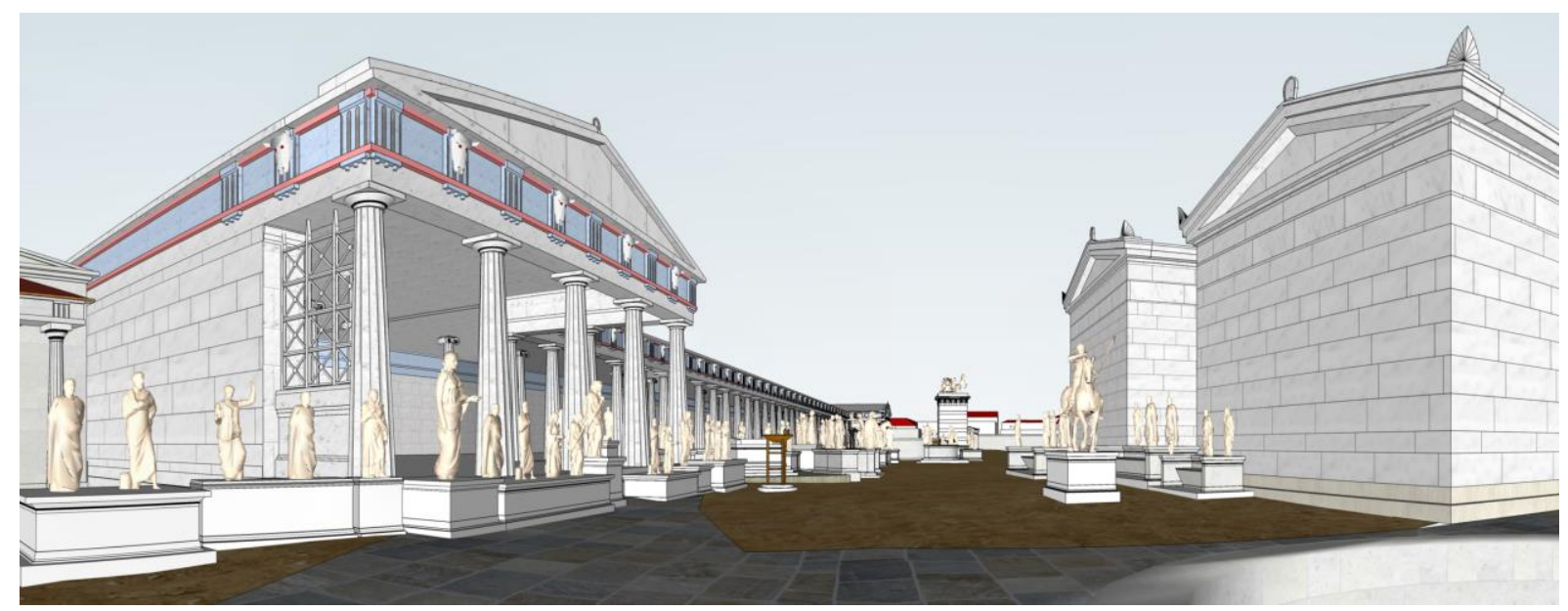

Figure 12. Portique d'Antigone (milieu du III' s. av. J.-C.) 
Du côté de l'entrée du bâtiment avait été aménagé un parvis monumental qui comportait une entrée à colonnes et, dans l'axe, un autel, sans doute l'Autel des rois que cite une inscription (figure 13). Il est évident que cet ensemble avait été conçu à la gloire de la dynastie qui avait libéré Athènes et Délos et fondé la confédération des Nésiôtes, regroupant la plupart des îles de l'Égée. Délos était une tribune pour les dynasties qui se disputaient l'Égée et se manifestaient dans l'espace et dans les cieux (colonnes).

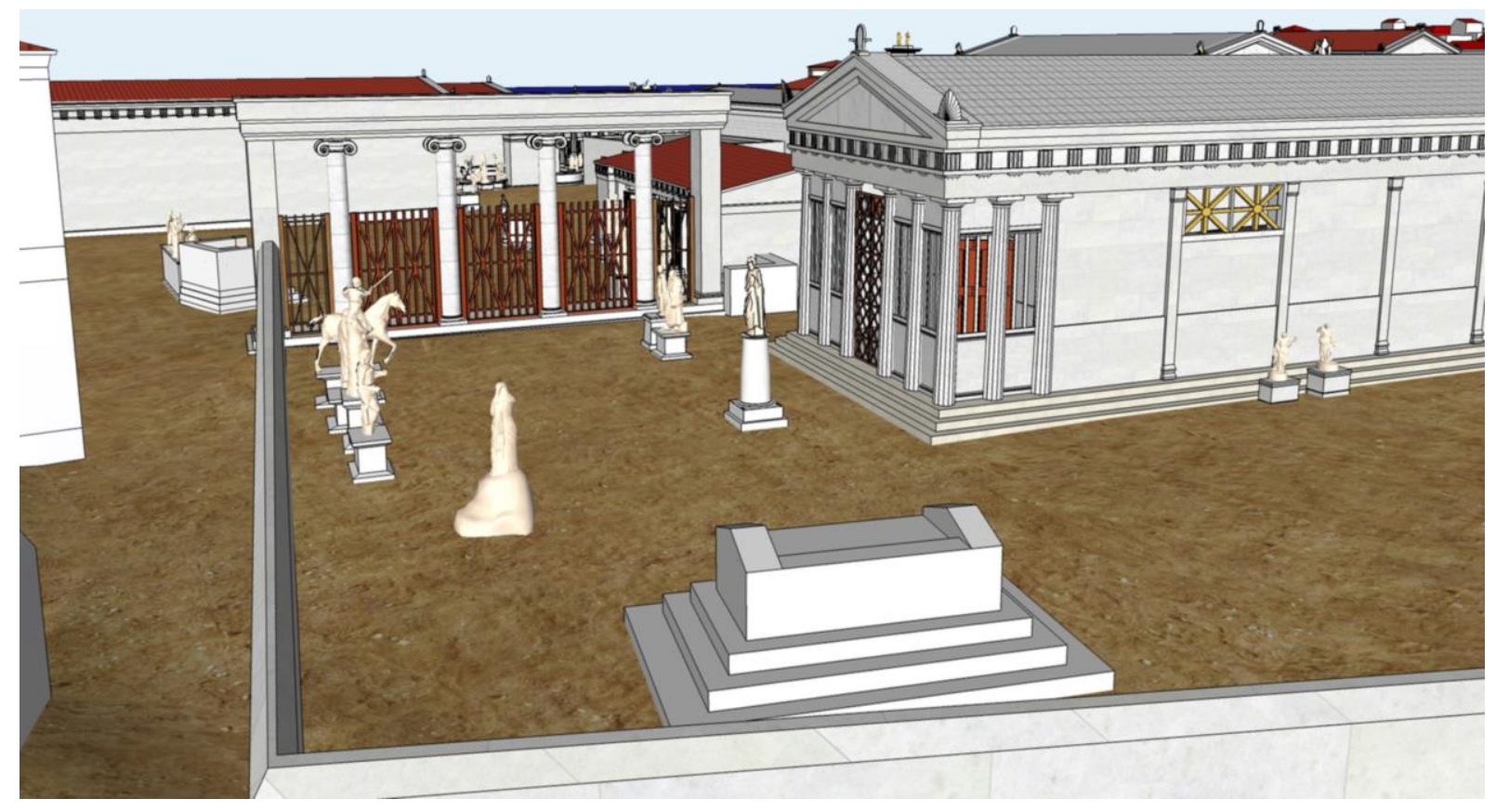

Figure 13. Esplanade devant le Monument des taureaux, vue de l'Est

Pendant la troisième période athénienne (167-88 av. J.-C.), les consécrations nouvelles, statues et décrets honorifiques, eurent du mal à trouver place au milieu des monuments plus anciens et l'accès des lieux de sacrifice, notamment de l'autel des Cornes, devait être difficile (figures 2-3). On assiste en effet à une explosion du nombre des statues : 101 mentions conservées pour cette période athénienne contre 33 pendant l'Indépendance. Les monuments honorifiques gardent les mêmes formes, mais c'est la sociologie des bénéficiaires qui change : si les rois sont toujours présents, les citoyens athéniens, magistrats ou particuliers regroupés en famille sur des exèdres, font leur entrée dans le Hiéron alors qu'ils étaient restés jusque-là à la périphérie (figure 14); la communauté locale décerne à un épimélète athénien un monument à deux colonnes, type d'offrande qui était jusque-là réservé aux rois, si bien que l'on peut parler d'un 'embourgeoisement' des bénéficiaires. Les espaces déliens subiront une nouvelle transformation après l'épisode de Mithridate, dont les troupes ravagent le sanctuaire en 88 av. J.-C., en s'ouvrant alors aux magistrats de Rome. 


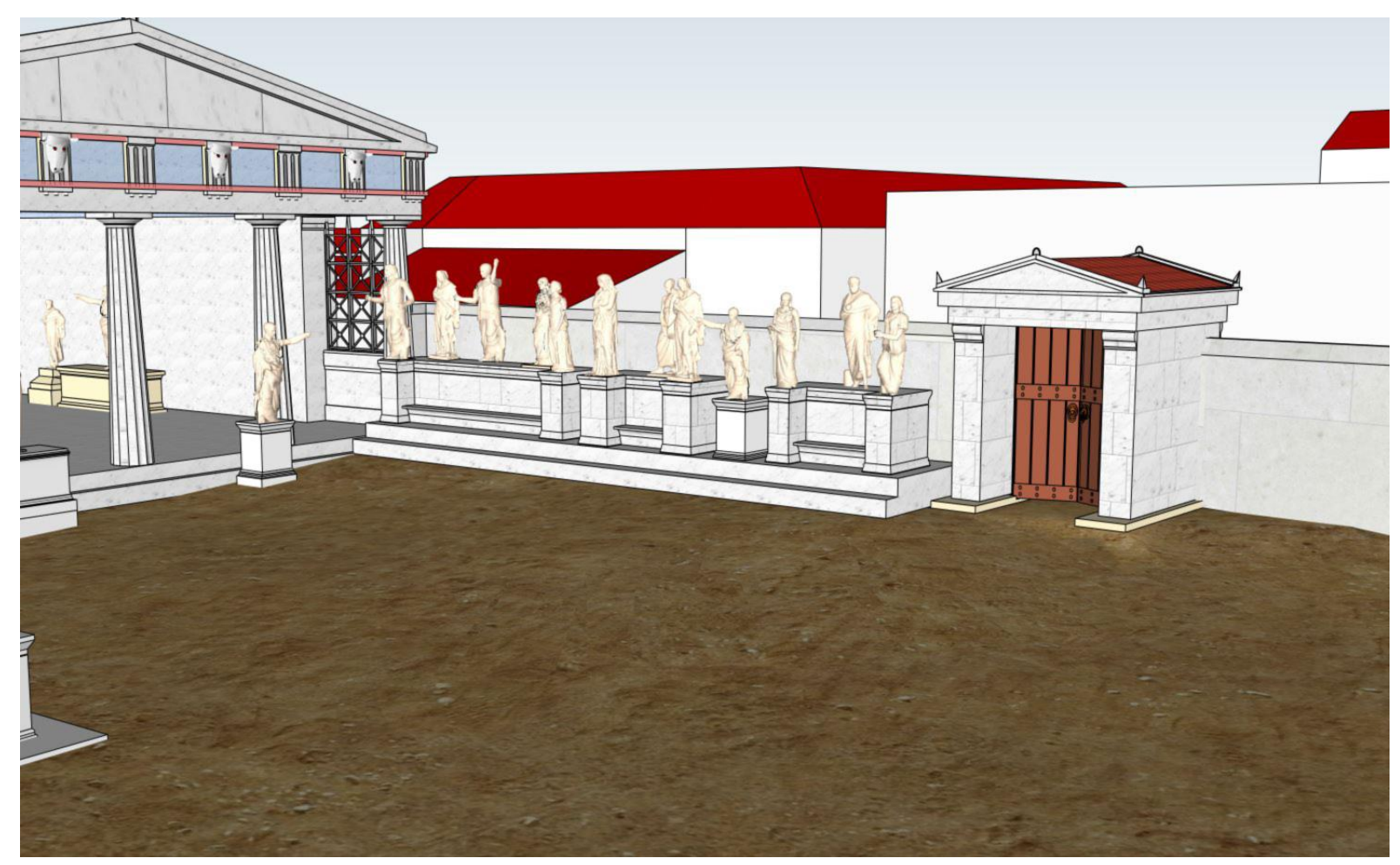

Figure 14. Entrée Nord-Est et extrémité Est du portique d'Antigone : exèdres familiaux d'Héphaistiôn de Mélité, Athénien (à gauche, 6 statues) et de Théodôra, fille de Kratéas de Téos et de Titus, fils de Satyrion d'Héracleia (extrème droite, 3 statues).

Les attaques piratiques de 88 et de 69 av. J.-C. ont marqué Délos et le sanctuaire. Les expressions employées par les auteurs anciens laissent penser que l'île fut entièrement dévastée et ruinée à cette occasion ${ }^{4}$. Toutefois, la situation décrite est sans doute exagérée et il convient de nuancer la portée de nos sources littéraires antiques: le Sanctuaire n'est pas devenu un champ de ruines du jour au lendemain. E. Lequéré a suivi ses transformations jusqu'au $I^{\mathrm{e}} \mathrm{s}$. de notre ère, et a tenté une cartographie de ces transformations, mais une restitution 3D n'est pas possible, par manque d'éléments suffisants sur l'état des bâtiments, ce qui n'est pas le cas des époques antérieures. Les effigies des empereurs firent leur apparition dans un espace sacré finalement peu transformé, mais dont la vie se concentra autour du Grand Temple, dans la partie Sud .

Le plan et les images 3D permettent de suivre les développements du sanctuaire sur plusieurs siècles. En 600 le sanctuaire est désespérément vide (figure 15) : on n'y trouve que deux temples, celui d'Apollon ou Oikos des Naxiens, premier temple d'Apollon, quoiqu'on en ait dit, celui d'Artémis, un petit temple anonyme et deux tombes des fameuses Vierges hyperboréennes, mortes à Délos en venant assister à la naissance d'Apollon.

\footnotetext{
${ }^{4}$ Strabon, X, 5, 4 : les généraux de Mithridate et le tyran qui avait soulevé Délos « la ruinèrent de fond en comble »; Pausanias, III, 23, 4 : « et il [= Ménophanès] rasa Délos elle-même jusqu'au sol ».
} 


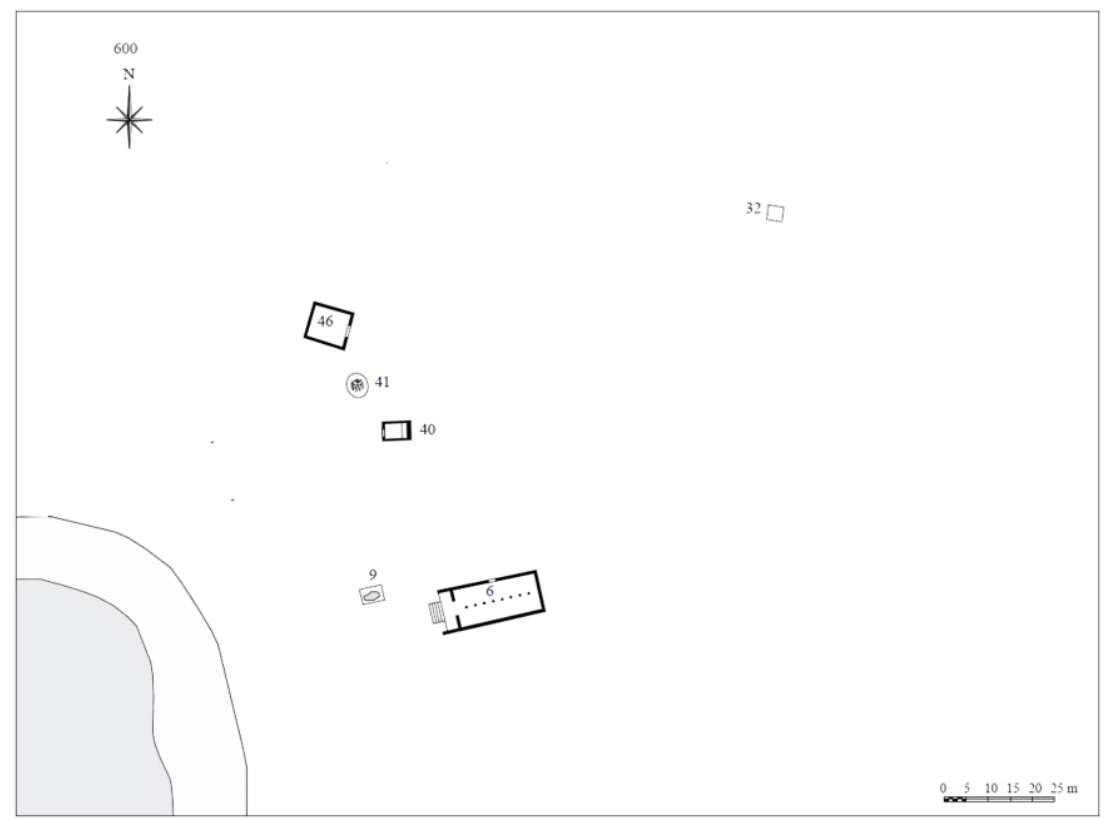

Figure 15. Sanctuaire d'Apollon en 600 av. J.-C.

Vers 500 en revanche le sanctuaire est structuré (figure 16) : les limites sont bien marquées par des périboles en dur, qui partagent l'espace sacré et distinguent clairement le domaine d'Artémis et celui d'Apollon qui ne cessera de croître. En 350 av. J.-C. (figure 17), le sanctuaire a pris la forme que l'on lui connaît dans les siècles postérieurs. Nous ne suivrons pas cette croissance puisque nous avons suffisamment commenté l'état final du $\mathrm{II}^{\mathrm{e}} / \mathrm{I}^{\mathrm{er}} \mathrm{s}$. av. J.-C. (figure 2).

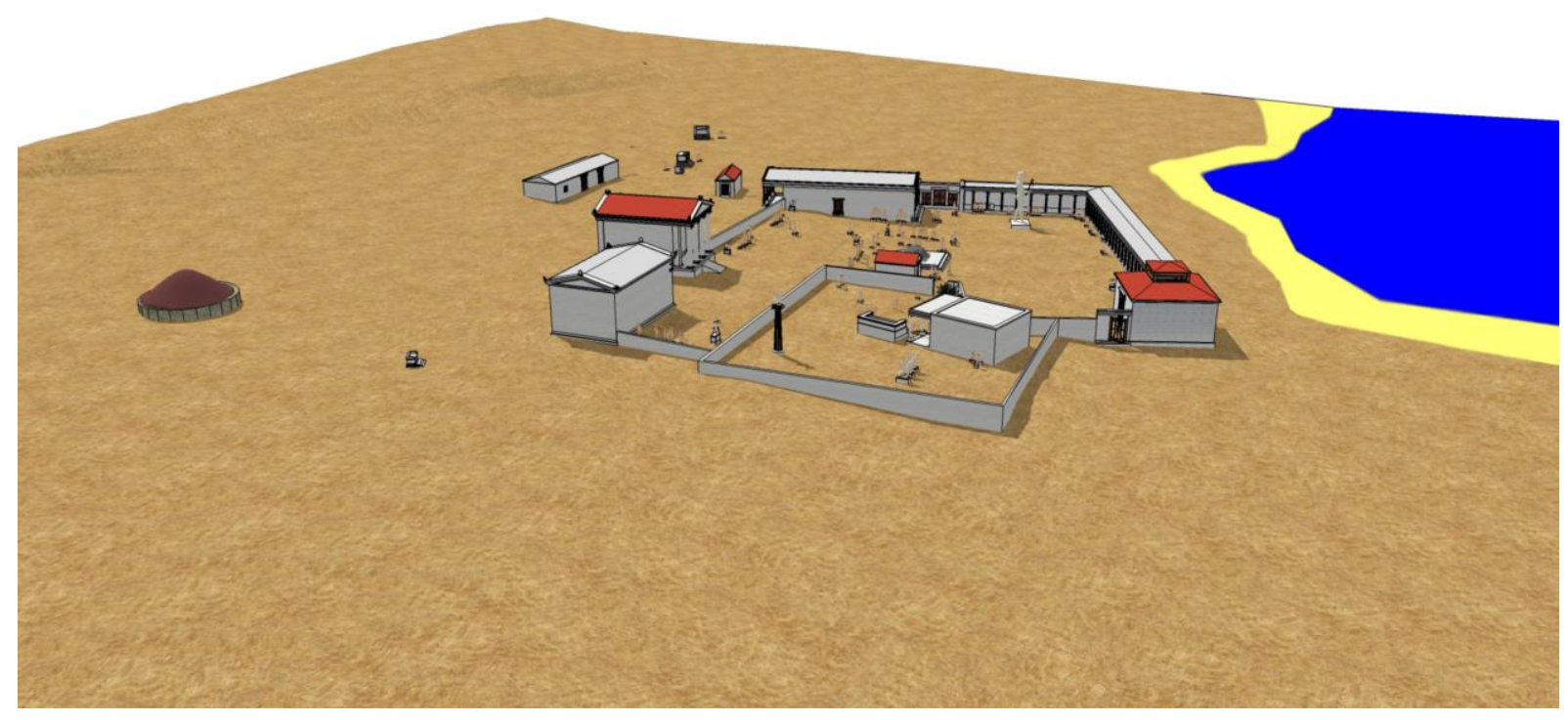

Figure 16. Sanctuaire d'Apollon en 500 av. J.-C., vue du Nord. 


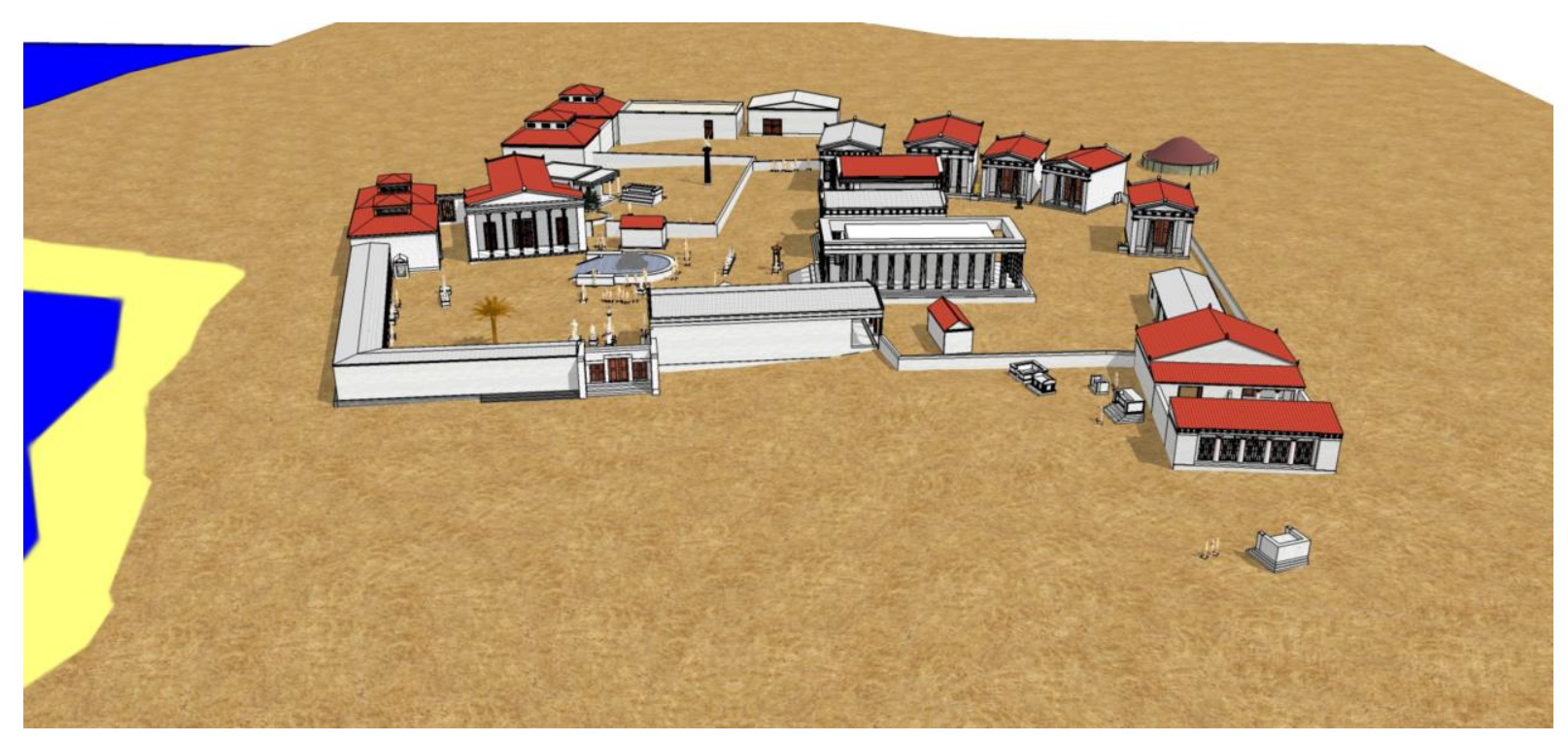

Figure 17. Sanctuaire d'Apollon vers 350 av. J.-C., vue du Sud.

Évoquons maintenant quelques problèmes liés à la représentation $3 \mathrm{D}$, telle que nous l'avons utilisée. Soulignons tout d'abord qu'il y a un véritable problème philosophique : jusqu'où peut-on ou doit-on aller dans la restitution? En fait les images que nous avons produites ne sont pas « moins » vraies que ne seraient des plans au sol. Ces images d'architectures restituées ne sont d'ailleurs pas en soi des nouveautés puisque dès le début des études déliennes de magnifiques restitutions ont été produites par les grands prix de Rome qui venaient traditionnellement sur les chantiers de Grèce. Il y a cependant une différence par rapport aux restitutions précédentes. Nous avons fait appel à une restitution tridimensionnelle numérique. Elle autorise des approches pour le moins inhabituelles en permettant de parcourir sous tous les angles le sanctuaire, non plus comme une série d'images mais comme une globalité en volume. Cette différence oblige à se poser différemment la question de l'espace.

Ajouter des statues sur des bases que l'on connaît ne déforme en rien, nous semble-t-il, la 'vérité'. D'un côté on pêche peut-être par excès, mais de l'autre certainement par défaut. On pourra bien sûr nous reprocher d'avoir choisi un peu arbitrairement les statues restituées. Mais elles étaient bien là où on les a placées et les empreintes donnent des indications. Pourquoi ne pas les exploiter jusqu'au bout?

Dans notre perspective de recherche, nous avons fait le choix d'utiliser la restitution tridimensionnelle en laissant de côté les aspects photo-réalistes pour nous orienter vers des images plus 'simples' du point de vue des textures et des éclairages. On pourra nous reprocher la simplicité des volumes pour l'architecture ou le manque de détails dans les textures des murs, des toits, ou encore la restitution des sols réalisée par un simple mappage simplifiant la réalité archéologique. Nous répondrons à ces critiques par des arguments dont certains sont d'ordre logistique et d'autres, plus importants, d'ordre méthodologique. D'abord, proposer des restitutions photo-réalistes suppose des moyens de calculs et des compétences informatiques dont un laboratoire public d'archéologie ne dispose que très rarement. Ensuite, le réalisme n'était pas l'option première choisie pour notre programme de recherche. Il s'agissait d'abord de travailler sur l'évolution dans le temps et dans l'espace de ce sanctuaire en positionnant dans les trois dimensions l'ensemble des données connues. Nous avons par exemple préféré restituer aussi fidèlement qu'il était possible les sols anciens et leur évolution et positionner de manière fidèle à la fois les 'grandes' et les 'petites' architectures sur la topographie antique. Cette démarche n’avait de fait jamais été entreprise.

La 3D n'est donc pas uniquement une façon attractive de présentation, elle permet de restituer des volumes et de visualiser la façon dont, à diverses époques, on a défini et utilisé des espaces. Elle démontre par exemple combien ces espaces étaient structurés dans une perspective d'inter-visibilité. N'oublions pas que le sanctuaire, s'il est pour nous un objet architectural est d'abord un lieu de culte, 
culte qui dans l'Antiquité faisait une place importante à la notion de déplacement dont témoignent les processions ; nous en avons donné dans ce texte quelques exemples. Cette maquette 3D constitue une nouvelle manière de voir le sanctuaire non plus comme un bel objet statique mais comme le support de circulations multiples. Elle présente un réel progrès en préparant des musées virtuels, qui éviteront des restitutions coûteuses et irréversibles. 